\title{
The role of memory and restricted context in repeated visual search
}

\author{
Melina A. KunAR \\ University of Warwick, Coventry, England \\ STEPHEN FLUSBERG \\ Stanford University, Stanford, California
}

AND

JEREMY M. WOLFE

Harvard Medical School, Boston, Massachusetts

and Brigham and Women's Hospital, Boston, Massachusetts

\begin{abstract}
Previous studies have shown that the efficiency of visual search does not improve when participants search through the same unchanging display for hundreds of trials (repeated search), even though the participants have a clear memory of the search display. In this article, we ask two important questions. First, why do participants not use memory to help search the repeated display? Second, can context be introduced so that participants are able to guide their attention to the relevant repeated items? Experiments 1-4 show that participants choose not to use a memory strategy because, under these conditions, repeated memory search is actually less efficient than repeated visual search, even though the latter task is in itself relatively inefficient. However, when the visual search task is given context, so that only a subset of the items are ever pertinent, participants can learn to restrict their attention to the relevant stimuli (Experiments 5 and 6).
\end{abstract}

To interact with the world, we often have to perform visual search tasks on a regular basis. For example, in our daily lives, we may try to find a car in a car park or a face in a crowd. Moreover, we create artificial visual search tasks of great social importance (e.g., finding a tumor in a mammogram or a hidden weapon in an airport baggage scan). In order to improve upon these tasks, we need to understand the mechanisms that occur when we search for an item. For this reason, researchers have investigated the process of visual search in the laboratory. Typically, participants are asked to respond to a prespecified target item among a variable number of competing distractor items. The reaction time (RT) taken to respond to the target item is used as a measurement of search speed. If we plot RT against the number of items in a display (the set size), we can plot the slope of the RT $\times$ set size function, which gives us a measure of search efficiency. If attention can be deployed readily to the target item, independently of the number of distractor items, we expect the search slope to be shallow, approaching $0 \mathrm{msec} /$ item. Efficient slopes are characteristic of feature searches, in which a target item can be separated from the distractors by means of a unique and salient feature (e.g., a red circle among green circles or a horizontal line among vertical; see Treisman \& Gelade, 1980).

In other visual search tasks, there is a cost to adding more distractor items to the search task, and slopes are sig- nificantly greater than zero. Such displays include search for a target that is made up of a conjunction of features (e.g., search for a red circle among green circles and red squares) or search for a target letter among heterogeneous distractor letters. In conjunction search tasks, in which some feature information can guide search (Wolfe, 1994; Wolfe, Cave, \& Franzel, 1989), slopes are intermediate in the efficiency they show, around 5-15 msec/item (e.g., Treisman \& Sato, 1990; Wolfe, 1998). Tasks such as letter search, lacking guiding features, tend to produce search slopes showing an inefficiency of around 30-50 msec/item (e.g., Kunar \& Humphreys, 2006; Theeuwes, Kramer, \& Atchley, 1998). These slopes are typical of tasks involving stimuli that are large enough to be identified in peripheral vision. If each item must be fixated, search is much less efficient, because efficiency is limited by the relatively slow rate of saccadic eye movements.

In earlier work, Wolfe, Klempen, and Dahlen (2000) investigated how the efficiency of a heterogeneous letter search task changed over time in two search conditions: a repeated search task and an unrepeated search task (see Figure 1). In this variant of a standard visual search task, a target probe was presented at the beginning of each trial to identify the target letter for each trial. In both conditions, participants had to indicate whether the target probe was present or absent from the search set on each trial. A target

M.A.Kunar, m.a.kunar@warwick.ac.uk 
Unrepeated
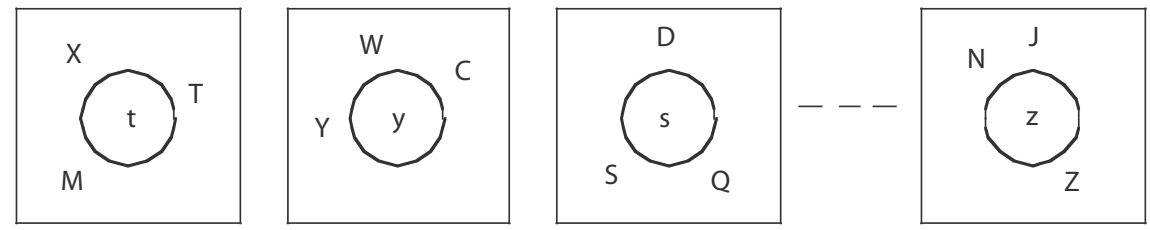

Repeated
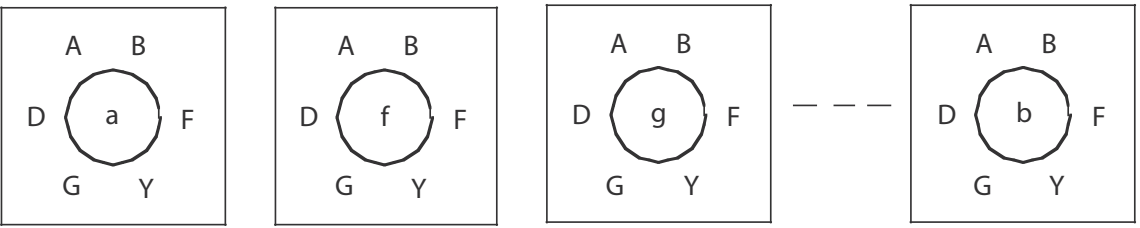

Memory
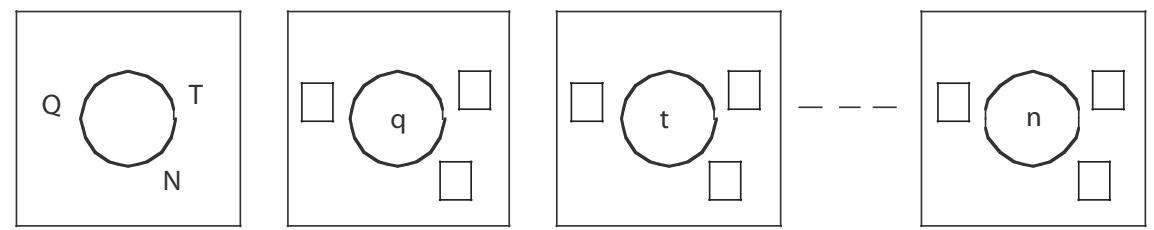

Time

Figure 1. Example of conditions in the repeated search experiments. The lowercase letter in the center circle indicates the target letter to be searched for on each trial. In the repeated search condition, the search display does not change throughout the condition. This is compared with a standard search, in which the display is changed from trial to trial. In the memory search task, participants memorize the display prior to the condition, after which the stimuli are removed from the display, or hidden.

was present on $50 \%$ of the trials. In the repeated search task, the search display remained the same throughout a block of trials. The identity and location of the search stimuli did not change, and the search stimuli did not disappear from the screen between trials. In the unrepeated search condition, the participants again had to search for a target probe that changed from trial to trial. However, here, the search display also changed from trial to trial. In the repeated search condition, familiarity with the display and/or repeated attention to specific letters in the display might be expected to lead to an improvement in search efficiency over time. One might imagine that less searching would be necessary on the 10th search for the same "F" at 3 o'clock in the same display. However, search in the repeated condition did not become more efficient over time but remained consistent at around $50 \mathrm{msec} /$ item even after 350 searches through the same, unchanging display. Repeated search efficiency was not significantly different from that in the unrepeated conditions.

This result seems counterintuitive in two ways. First, observers come to learn and remember that " $F$ " was at 3 o'clock. Why did they not use this memory to speed search when the target was an "F"? Second, one would think that repeated search would be an efficient way to search. In the real world, when a scene becomes familiar, we reduce the effective set size and search only where we learn that things are likely to be. Why do we not see this apparent reduction of potential target locations in repeated search tasks? This article addresses these two puzzles.

The first question was actually made more puzzling by the original Wolfe et al. (2000) work. The article included a memory search condition, in which participants committed the letter display to memory. The search stimuli were removed, and the participants indicated whether the target probe item was present or absent from the memorized search display. In this case, repeated search from memory did become more efficient. This replicates an established result in memory search. Previous work on memory search has shown that initial search slopes for a task such as this lie in the range of 20-50 msec/item (see Sternberg, 1975, for a review). With repetition, these search slopes show a greater efficiency and, in some instances, asymptote at around 0 msec/item (e.g., Logan, 1992). These memory searches are said to have become automatized. Schneider and Shiffrin (1977) explained this as a form of consistent mapping in which a target-present response is mapped to one set of letters (if the target is present) and a target-absent response is mapped to another set (if it is absent). Given that memory search could apparently proceed efficiently, why did the observers not use that memory to guide the visual search?

Wolfe et al. (2000) argued that vision was given priority over memory in a visual search task. Oliva, Wolfe, and 
Arsenio (2004) asked whether inefficient visual search had a mandatory priority over efficient memory search. They found that this was not the case. In their research, in which they used a variation of the repeated search task in which the participants searched a fixed display but that display was larger than the current field of view (dubbed a panoramic search display), the participants were able to search from memory once they had been extensively trained to do so. On any one trial, the participants viewed a subset of the whole visual display as a viewing window panned back and forth over a larger scene. During a series of experiments, the participants could be asked to respond to (1) items that were present in the display and also currently visible, (2) items that were present in the overall display but were currently hidden from view (i.e., in a section that had previously been seen but was not now part of the panned visible section; here, they had to use their memory), or (3) items that were absent from the entire display. The results showed that the participants performed inefficient visual searches until "persuaded," by extensive experience with the hidden stimuli, that they could rely on memory. Thus, it is possible to use memory in the presence of a visual stimulus; however, visual search seems to be the preferred mode. Oliva et al. argued that the participants made a "pragmatic choice" between vision and memory and suggested that they were biased to perform a visual search task over a memory one, even if visual search was less efficient than memory search.

Still, if memory search really was more efficient than visual search, why do participants initially choose to use the less efficient strategy? In fact, the repeated search task does not demand that observers choose between visually searching for the target or responding from memory alone. Common sense would seem to tell observers to remember where the target was and then to visually confirm that memory. In Experiments 1-4, we solve this mystery by showing that memory search is actually less efficient than visual search. In the previous work of Wolfe et al. (2000) and Oliva et al. (2004), observers made a choice, but it was not a choice between two modes of search. The automatized memory search in Wolfe et al. (2000) was not a search task so much as it was an efficient responsemapping task. The observers learned to associate one set of probe letters with one response key and another set with the other response (see Schneider \& Shiffrin, 1977). In the present experiments, we made the memory task more like the visual task by making both of them localization tasks. Participants had to point and click on an individual target location. This change had several benefits. First, it made both tasks a little more realistic. In the real world, search tasks are typically carried out in order to direct action toward a target. One does not generally search for the milk merely to confirm that it is still in the refrigerator. Second, the method ensured that each search stimulus required a different response. Please note that in all the tasks, the number of stimulus-to-response (S-R) mappings was equal. This is important, since the Hick-Hyman law states that increasing the number of response alternatives increases the time taken to respond (Hick, 1952; Hyman, 1953). Finally, it made it more likely that the participants were performing similar tasks in the memory and the visual search conditions. With this method, Experiment 1 showed that memory search was both inefficient and slow, as compared with a visual search task in which there were six possible responses. Experiment 2 showed that memory search was inefficient and slow if there were up to six multiple response types, as compared with a two-alternative forced choice (2AFC) task. The primary conclusion of this article is that participants do not use memory to guide inefficient visual search processes in the repeated search task, because the memory that could do the guiding is even less efficient. Experiments 3 and 4 showed that even after extensive training, of the sort used in Oliva et al. (2004), memory search remained inefficient.

Turning to the second question posed above: Given that we can learn to restrict our attention to potential target locations in most real-world searches, why do we apparently fail to restrict our attention in the repeated search task? The answer lies in the structure of the tasks. As was mentioned above, in the real world, when a scene becomes familiar, we reduce the effective set size. The number of plausible locations for an object declines, and the number of potential target items may also decline. Initially, the cat could be anywhere. Eventually, you learn that he has three favorite spots, and you restrict your initial search to those locations. In the repeated search task, in contrast, the numbers of targets and their locations never change. If the observer is looking for these six letters in these six locations on Trial 1, he or she is looking for the same six letters in the same six locations several hundred trials later. Every item in the display is, at one point or another, relevant and, thus, searched. This turns out to be critical. In Experiments 5 and 6, we modified the repeated search task to allow the observers to learn that targets can appear in some locations, but not in others. Under these conditions, observers can use memory to restrict their attention to a subset of target locations and, thus, use this to guide their visual attention.

\section{EXPERIMENT 1}

In Experiment 1, we investigated whether memory search in a repeated search task would be more efficient than visual search when the stimuli could not be consistently mapped to two response types (e.g., is the target present or absent?). In this experiment, rather than making the usual $2 \mathrm{AFC}$ response, the participants had to use the mouse to point to the location of the target item in both the repeated visual search and the memory search conditions. This made sure that the mapping of test probe to response was the same in the visual and the memory search versions of the task.

\section{Method}

Participants. Twelve individuals between the ages of 18 and 55 years served as participants. Each participant passed the Ishihara test for color blindness and had normal or corrected-to-normal vision. All the participants gave informed consent and were paid for their time.

Apparatus and Stimuli. The experiment was conducted on a Macintosh computer using MatLab Software with the PsychToolbox (Brainard, 1997; Pelli, 1997). The search stimuli consisted of three or six uppercase letters of the English alphabet. All the letters were white and appeared on a black background. The dimensions of the 
letters ranged from $0.3^{\circ}$ to $1^{\circ}$ in width by $1^{\circ}$ to $1.3^{\circ}$ in height (depending on the letter), and each letter was positioned at a distance of $4^{\circ}$ from the center. The target probe was a lowercase letter $\left(0.1^{\circ}-0.6^{\circ}\right.$ in width, $0.5^{\circ}-1^{\circ}$ in height) presented at the center of the screen within a circle with a diameter of $1.5^{\circ}$.

Procedure. There were three conditions: (1) a repeated visual search task, (2) an unrepeated visual search task, and (3) a memory search task. At the start of each trial, a cursor appeared in the center of the circle. In all the conditions, the participants were instructed to move the cursor and to click on the location of the uppercase target letter that corresponded to the lowercase cue. The lowercase cue appeared in the central circle and changed from trial to trial; however, it was always a letter that was present in the outer display (i.e., there were no target-absent trials). In the repeated search condition, the display did not change and remained visible throughout the experiment. In the unrepeated search condition, the display letters changed from trial to trial. In the memory search condition, the participants were instructed to memorize the positions and identities of the display letters prior to the task. During the search task, the letters were removed and replaced by white-framed black boxes. The participants were asked to click on the box corresponding to the now hidden target letter. In all the conditions, the participants were asked to respond as quickly and as accurately as possible. In order to be counted as a correct response, with all set sizes, the participants had to make sure that they clicked within the outlined box (of dimensions $1.3^{\circ} \times 1.8^{\circ}$ ). Furthermore, they were encouraged to move the mouse cursor directly to the target. For each condition, each participant completed one block of trials with set size 3 and one with set size 6 . Each block consisted of 20 practice trials and 500 experimental trials, which were divided into 10 epochs of 50 trials for analysis. The order of the blocks was randomized. Figure 1 shows example displays for each condition.

\section{Results and Discussion}

Figure 2A shows RT as a function of epoch for each combination of condition and set size, and Figure 2B shows overall RT as a function of set size. At each epoch, search slope is computed from the average RTs for set sizes 3 and 6. Those slopes, the critical measures of search efficiency, are shown in Figure 3. The results are clear. Replicating the original repeated visual search results, search remained inefficient after 500 trials - just as inefficient as unrepeated search. Moreover, with this change in method, repeated memory search was markedly less efficient than visual search, even after 500 trials.

Overall, error rates were low (less than 1\%). There was a main effect of condition: The participants made more errors in the memory condition than in the repeated and unrepeated search conditions $[F(2,22)=4.2, p<.05]$. However, none of the other main effects or interactions proved reliable. Since the error rates suggest that there was no speed-accuracy trade-off, we will not discuss them further and, instead, will concentrate on RT and slope analysis. RTs below $200 \mathrm{msec}$ and above $4,000 \mathrm{msec}$ were removed from analysis. This led to the removal of fewer than $1 \%$ of the data. There are many main effects and interactions that could be reported in this experiment. For the sake of simplicity and brevity, we will focus on those analyses that are relevant to the questions addressed in this article.

There was no effect of epoch on any of the conditions. Overall, RTs did not decrease with trial number in the repeated condition $[F(9,99)=0.7$, n.s., and $F(9,99)=1.5$, n.s., for set sizes 3 and 6 , respectively], the unrepeated condition $[F(9,99)=1.1$, n.s., and $F(9,99)=0.6$, n.s., for set sizes 3 and 6 , respectively], or the memory condition $[F(9,99)=0.7$, n.s., and $F(9,99)=1.7$, n.s., for set sizes 3 and 6 , respectively].

There were significant RT differences between conditions. Comparing the repeated and the unrepeated search conditions, we see that RTs in the repeated condition were shorter than those in the unrepeated condition $[F(1,11)=$ $17.5, p<.01]$. This occurred for both set size 3 [98 msec; $F(1,11)=7.4, p<.05]$ and set size $6[106 \mathrm{msec} ; F(1,11)=$ $20.2, p<.01]$. This replicates the findings of both Wolfe et al. (2000) and Oliva et al. (2004). The overall slowing of responses between repeated and unrepeated tasks could have been due to a number of factors. First, the items in the repeated condition were consistently mapped to a response, whereas the items in the unrepeated condition were not. Inconsistent-mapping tasks are known to be slower than consistent-mapping tasks (Schneider \& Shiffrin, 1977). Second, RTs in the unrepeated search condition might have been slowed by a cost associated with front-end perceptual processing of a new visual display on each trial.

Overall, RTs in the memory search task were longer than those in the repeated search task $[F(1,11)=7.1, p<$ $.05]$. As can be seen in Figures 2 and 3, this was driven by a large difference at set size $6[148 \mathrm{msec} ; F(1,11)=$ $12.3, p<.01]$. There was no reliable difference at set size 3 $[F(1,11)=0.4$, n.s. $]$. None of the condition $\times$ epoch interactions proved to be significant. This pattern of data was different from that found by Wolfe et al. (2000). In those experiments, using a $2 \mathrm{AFC}$ response task, RTs in the memory search task were shorter than those in the repeated search task and the RTs in the memory task interacted with epoch. The present data show that when a target localization task was used, the pattern of results was reversed.

Turning to the slope data, slopes in the memory condition were substantially larger than those in the repeated search condition $[F(1,11)=7.5, p<.05]$. Furthermore, search slopes in the memory condition did not show a greater efficiency over time $[F(9,99)=0.4$, n.s. $]$. This pattern was the direct opposite of that found in the 2AFC repeated search tasks, where performance on that memory task did improve over time and did become more efficient than the repeated search (Wolfe et al., 2000). The visual search data reported here are similar to those from the original $2 \mathrm{AFC}$ repeated search findings (Wolfe et al., 2000). Search efficiency did not improve over epoch in either the repeated search or the unrepeated search condition $[F(9,99)=0.5$, n.s., and $F(9,99)=0.5$, n.s., respectively], and there was no overall difference between the search slopes in the repeated search condition and those in the unrepeated search condition $[F(1,11)=0.0$, n.s.].

The results make a number of interesting points. First, these data serve as a replication of the basic repeated visual search results reported by Wolfe et al. (2000). Search slopes in the repeated search task did not differ from those in the unrepeated search task and did not decrease over time. The participants were unable to perform an efficient letter search even after searching for the same letters in the same, unchanging display for hundreds of trials. Second and more important, the data provide a response as to why the participants chose not to use memory when faced with 
A

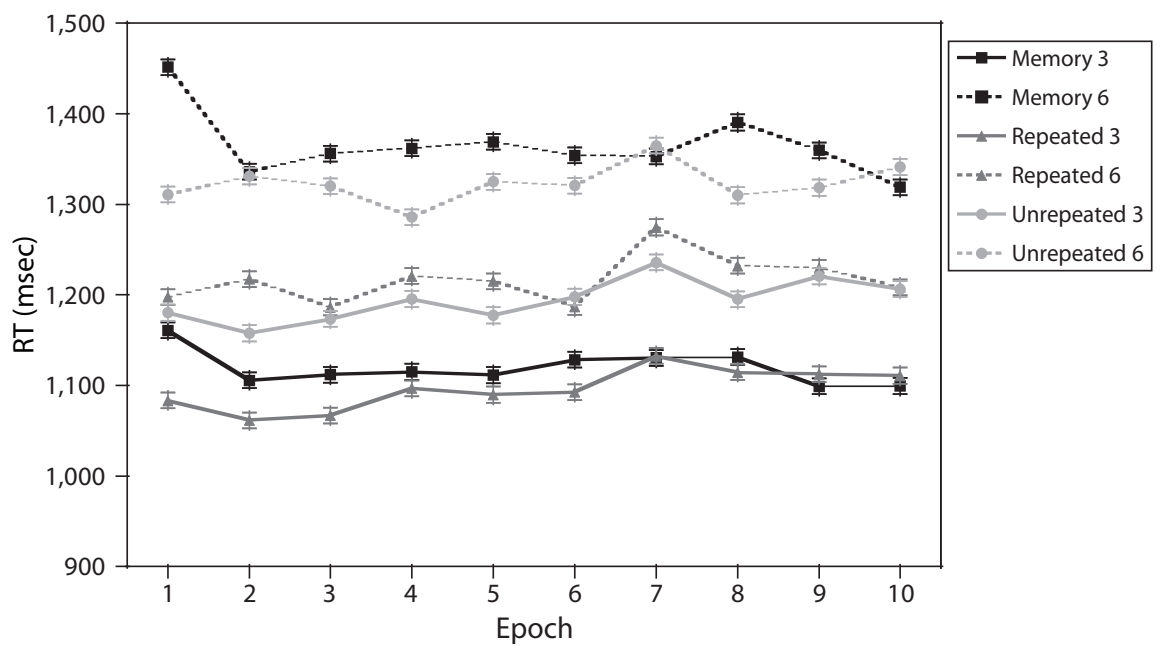

B

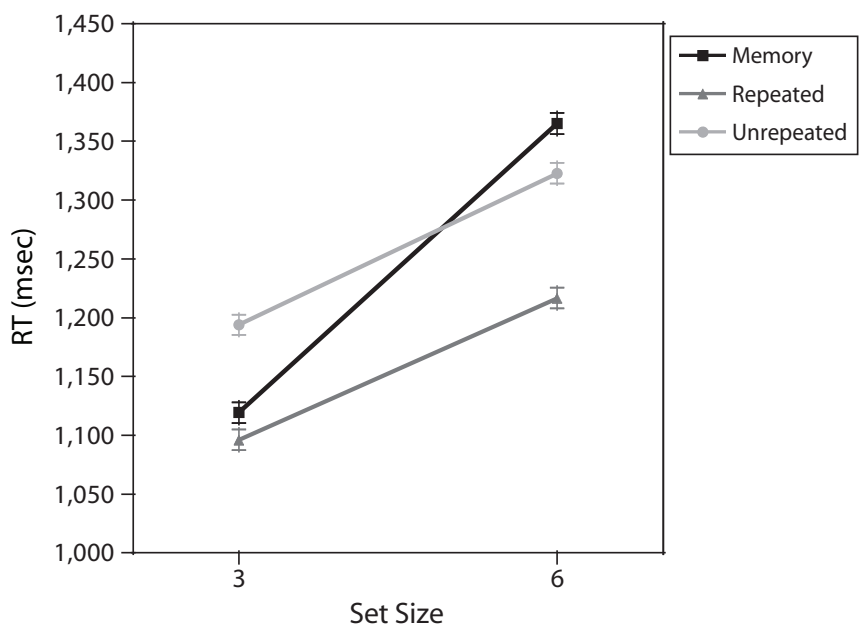

Figure 2. (A) Mean reaction times (RTs) across epochs for each condition in Experiment 1. (B) Overall mean RT as a function of set size for each condition in Experiment 1.

a repeated search display. The answer is that guidance by memory search did not occur because the memory search was less efficient than visual search.

Why did this experiment fail to show the improvement in efficiency in memory search that was found in Wolfe et al. (2000)? It is not simply that localization tasks cannot be efficient. For example, Logan, Taylor, and Etherton (1999) found that location could be encoded during automatization. In their work, they trained participants to respond to sets of visual stimuli, so that RTs became shorter with practice. In a test phase, if the location of the items changed, Logan et al. found that the time taken to respond to each stimulus increased, suggesting that the consistent mapping of location to response could be encoded. Furthermore, in a baseline study, we ran a $2 \mathrm{AFC}$ repeated search localization task, in which participants had to respond to whether the target was to the left or the right of the central fixation probe. The experiment included repeated search, unrepeated search, and memory search conditions. The results showed that a $2 \mathrm{AFC}$ localization version of a memory search task was more efficient than a $2 \mathrm{AFC}$ localization version of a repeated visual search $\operatorname{task}[F(1,15)=4.5, p=.05]$. These memory results mimicked those for the $2 \mathrm{AFC}$ present/absent task found in the memory search conditions in Wolfe et al. (2000) and Oliva et al. (2004). Simply introducing a spatial element to the task did not cause the pattern of the results to change.

Instead, we propose that the earlier apparent advantage for memory search was actually the result of a change in the task that the observers were performing. Wolfe et al. (2000) wanted observers to search either the visual stimulus or the memory set. Instead, the observers learned to efficiently use the response mappings of two different probe types (i.e., present or absent) to two different responses. In contrast, our Experiment 1 forced observers to continue to search, by eliminating the $2 \mathrm{AFC}$ response option. Under 


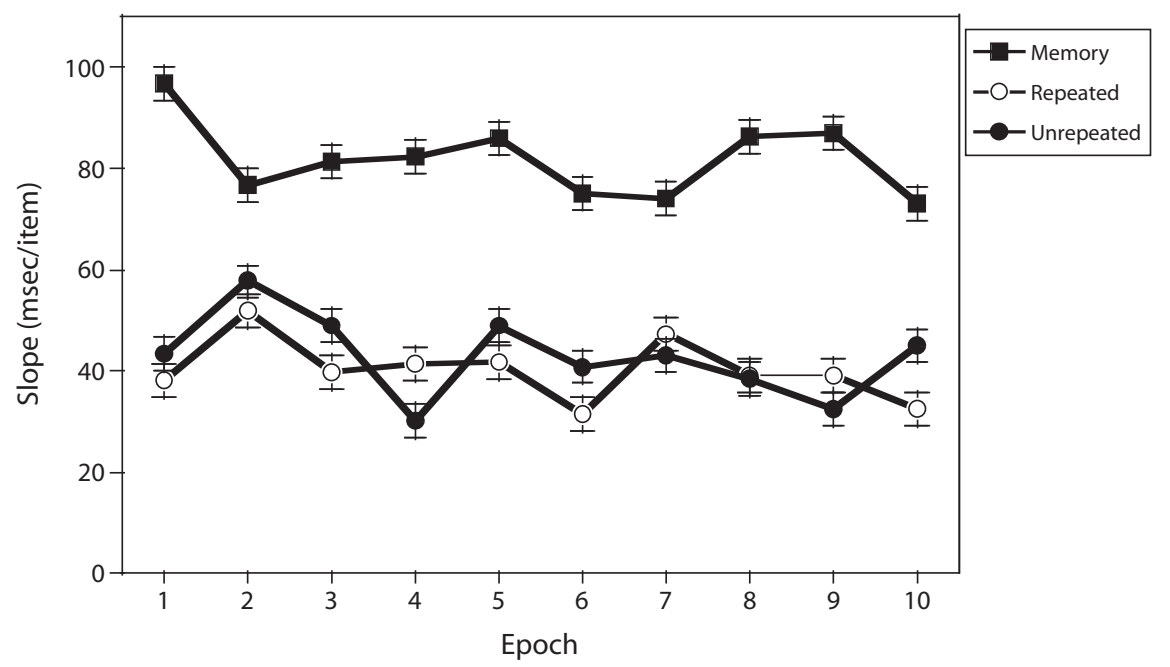

Figure 3. Mean search slope (milliseconds/item) across epochs for each condition in Experiment 1.

these circumstances, we found that efficiency did not improve over the course of 500 trials.

\section{EXPERIMENT 2}

The analysis of Experiment 1 relies on the assumption that memory tasks with a small number of response alternatives can become automatic, whereas tasks with a larger repertoire of possible responses do not. This hypothesis was tested in Experiment 2 by directly comparing a $2 \mathrm{AFC}$ and a localization version of a memory search task.

\section{Method}

Participants. Fourteen individuals between the ages of 18 and 55 years served as participants. Each participant passed the Ishihara test for color blindness and had normal or corrected-to-normal vision. All the participants gave informed consent and were paid for their time.

Apparatus and Stimuli. The apparatus and stimuli were similar to those in the memory search condition in Experiment 1, except that here, the target probe appeared directly above the central circle, so that it was not partially occluded by the initial cursor that appeared within the central circle. Please note that any occlusion in Experiment 1 was minimal and did not affect the pattern of results.

Procedure. There were two conditions in this experiment: (1) a localization condition in which the participants clicked on the remembered location of the target and (2) a $2 \mathrm{AFC}$ condition in which the participants determined whether the target was present or absent. In both conditions, the participants were instructed to memorize the positions of the capital letters prior to a block of trials. During the memory search, the capital letters were removed and replaced by white-framed black boxes. The localization task was similar to the task in the memory search condition in Experiment 1. The appearance of the display of the 2AFC present/absent task was similar to that of the localization task. However, the target probe corresponded only to one of the capital letters on approximately half of the trials. On the remaining trials, the target probe was selected from a group of $N$ letters that were not present, where $N$ equaled the set size. On each trial, the participants pressed the letter "l" if the target was present and the letter " $a$ " if the target was absent.

For each condition, the participants completed five blocks of trials, one each for set sizes 2, 3, 4, 5, and 6. Each block consisted of
350 trials, which were divided into seven epochs of 50 trials. The order of the blocks was randomized.

\section{Results and Discussion}

In Figure 4, the slopes for the 2AFC and the localization tasks are compared. Curiously, performance on the $2 \mathrm{AFC}$ memory task did not become particularly efficient. However, it is clear that search was more efficient in the 2AFC version than in the localization version, although the only difference between the tasks was the mode of response.

Overall, error rates were low (5.8\% in the 2AFC memory condition and $1.9 \%$ in the memory location condition). Since the error rates suggest that there was no speedaccuracy trade-off, we will not discuss them further and, instead, will concentrate on RT and slope analysis. RTs below $200 \mathrm{msec}$ and above $4,000 \mathrm{msec}$ were eliminated. This led to the removal of $1 \%$ of the data.

The RTs from the localization condition increased with set size $[F(4,52)=18.7, p<.01]$. There was also an overall effect of epoch. In fact, in this case, RTs increased modestly with time $[F(6,78)=3.1, p<.01]$. Although there appears to be an initial drop in slope in the localization conditions (Figure 4), there was no reliable set size $\times$ epoch interaction $[F(24,312)=1.1$, n.s.] or any reliable effect of epoch on search slopes $[F(6,78)=1.0$, n.s.]. These data replicate those in Experiment 1. ${ }^{1}$

Turning to the target-present trials in the $2 \mathrm{AFC}$ condition, there was a main effect of set size $[F(4,52)=18.4$, $p<.01]$ and epoch $[F(6,78)=7.8, p<.01]$. In general, RTs increased with set size and decreased with epoch. This decrease in RTs across epochs was more pronounced at higher set sizes, as indicated by a reliable set size $\times$ epoch interaction $[F(24,312)=2.0, p<.01]$. The same pattern occurred for target-absent trials. In contrast to the localization condition, search slopes show a greater efficiency over time. This occurred for both target-present trials $[F(6,78)=5.0, p<.01]$ and target-absent trials 


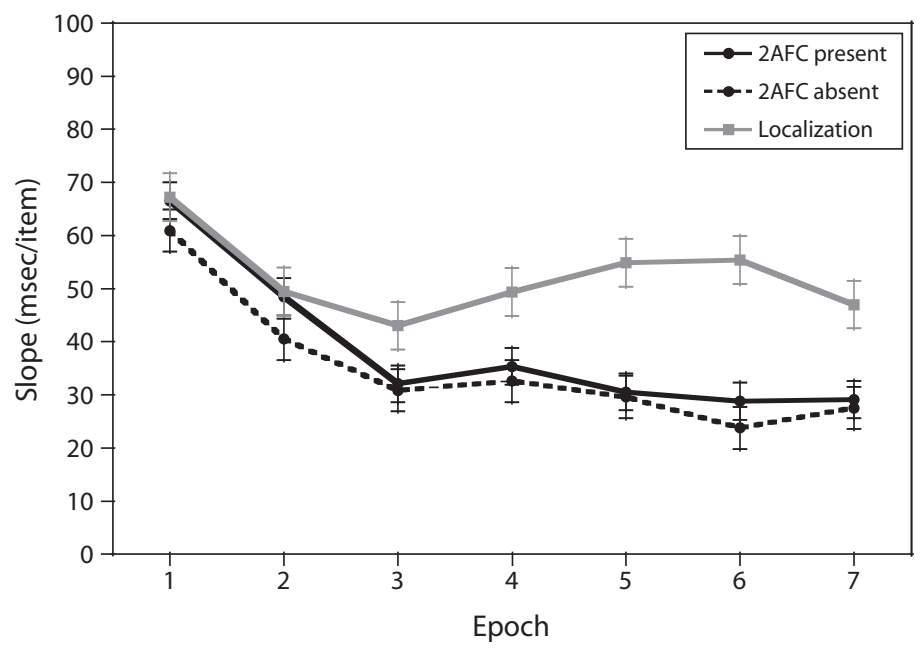

Figure 4. Mean search slope (in milliseconds/item) for repeated memory search across epochs for each condition in Experiment 2.2AFC, two-alternative forced choice.

$[F(6,78)=3.3, p<.01]$. Thus, the data from the $2 \mathrm{AFC}$ condition mirrors that in Wolfe et al. (2000), although the slopes for the present/absent condition asymptote at a surprisingly inefficient $25-30 \mathrm{msec} /$ item.

Overall, the results are consistent with the hypothesis that performance on $2 \mathrm{AFC}$ tasks becomes more efficient with extensive practice, whereas that on localization tasks, requiring a mapping of more than two responses, does not become more efficient. This, in turn, supports the account of the memory search advantage in the Wolfe et al. (2000) data. The well-practiced 2AFC task became a responsemapping problem, whereas the visual search task remained a search/localization task. We would suggest that in the panorama experiments in Oliva et al. (2004), visual search became more efficient with practice because observers learned to treat the repeated visual search task like a $2 \mathrm{AFC}$ memory task even when the targets were intermittently visible. In Experiment 3, we examined the effects of similar training on the localization task.

\section{EXPERIMENT 3}

In Experiment 3, the observers were trained on a mixed visual and memory search localization task in order to determine whether they could learn to perform an efficient repeated memory search with a localization response.

\section{Method}

Participants. Thirteen individuals between the ages of 18 and 55 years served as participants. Each participant passed the Ishihara test for color blindness and had normal or corrected-to-normal vision. All the participants gave informed consent and were paid for their time.

Apparatus and Stimuli. The apparatus and stimuli were identical to those in Experiment 2.

Procedure. There were three conditions: a pure memory search control condition and two training conditions - memory search with repeated visual search training and memory search with mixed vi- sual and memory training (see Figure 5 for example displays of the latter condition). In all the conditions the participants were asked to click on the location of the target. For each condition, the participants completed two blocks of trials, one each for set sizes 3 and 6. Each block consisted of 500 trials, which were divided into 10 epochs of 50 trials. The order of the blocks was randomized. The pure memory search condition was identical to the localization condition in Experiment 2 . The participants memorized the display and proceeded to search through three or six hidden stimuli for 500 trials. In the visual training condition, the search stimuli were visible for the first 150 trials (the initial training phase). After this, they were removed and replaced by white-framed black boxes. The participants completed the remaining 350 trials from memory. In the mixed training condition, the participants were instructed to memorize a search display prior to the task. During the initial training phase, for set size 6 , three letters of the search display remained visible while the other three letters were replaced by black white-framed boxes, whereas in set size 3 , one letter remained visible while the other two letters were replaced by black white-framed boxes. After 150 trials (the posttraining phase), all the letters were replaced by white-framed black boxes so that none of the stimuli were visible. As in the other conditions, the participants had to respond from memory for the remaining 350 trials.

\section{Results and Discussion}

Figures 6 and 7 show the RTs and search slopes, respectively, for all the conditions in Experiment 3. The results were clear. There was no benefit from either training regime. After training, all three versions of localization memory search were inefficient and did not improve over hundreds of trials.

Overall, error rates were low (less than $1.5 \%$ ). There was a main effect of set size, with the observers making more errors at the larger set size $[F(1,11)=6.0, p<.05]$, and a main effect of epoch, reflecting an increase in errors after training ended $[F(9,99)=2.9, p<.01]$. There was also a reliable condition $\times$ epoch interaction $[F(18,198)=2.2$, $p<.01]$ and a set size $\times$ epoch interaction $[F(9,99)=2.6$, $p<.01]$. However, since the error rates suggest that there was no speed-accuracy trade-off, we will not discuss errors further and, instead, will concentrate on RT and slope 
Training: Set Size 3

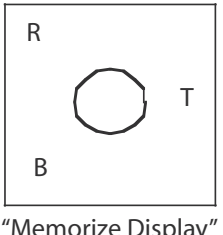

Training: Set Size 6

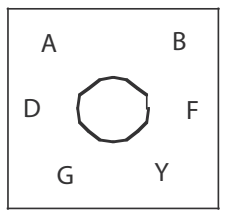

"Memorize Display"

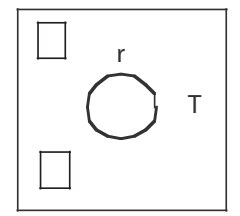

Trial 1

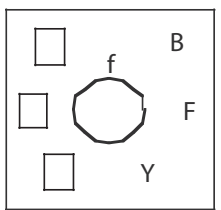

Trial 1

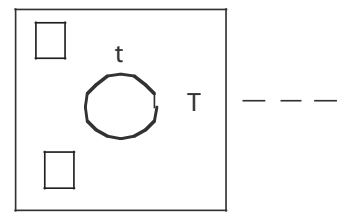

Trial 2

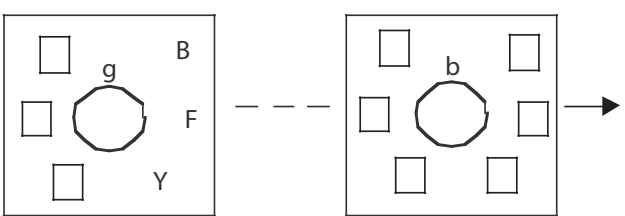

Trial 2

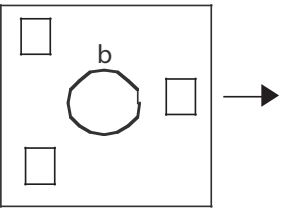

Trial 150

Trial 150

Figure 5. Example displays of the mixed training condition in Experiment 3.

analysis. RTs below $200 \mathrm{msec}$ and above $4,000 \mathrm{msec}$ were eliminated. This led to the removal of $1 \%$ of the data.

For each condition, the data were split up into the initial training phase (Epochs 1-3) and the posttraining phase (Epochs 4-10) for analysis. During the initial training period, there were reliable differences between the conditions in $\mathrm{RT}[F(2,22)=9.6, p<.01]$ and slope $[F(2,22)=$ $11.0, p<.01]$. Performance in the training conditions, with some or all items visible, were faster and more efficient than that in the pure memory search condition. After training (Epochs 4-10), there were no significant differences in RT $[F(2,22)=0.2$, n.s. $]$ or slope $[F(2,22)=$ 0.4 , n.s.]. Comparing the end of the training period with the beginning of the posttraining period, RTs at set size 6 become longer in both the visual and the mixed training conditions $[t(11)=4.4, p<.01$, and $t(11)=6.8, p<.01$, respectively]. There was no change in RTs at set size 3 . Slopes become steeper in both the visual and the mixed training conditions $[t(11)=4.2, p<.01$, and $t(11)=4.7$, $p<.01$, respectively].

Hillstrom and Logan (1998) suggested that mechanisms in memory search were a subset of those in visual search. Therefore, training in visual search led to improved performance in memory. At first glance, our work seems to contradict that of Hillstrom and Logan. In our Experiment 3, memory search did not become more efficient over time, even after visual or partial visual training. The critical difference appears to be the use of the localization task. If the memory task cannot be reduced to a $2 \mathrm{AFC}$ task, it is highly inefficient even after 500 trials of search through the same memory set.

Perhaps memory search in the training condition in Experiment 3 did not become more efficient because the participants were not trained hard enough. In the initial training phase, a given item was either visible or hidden. However, in the posttraining phase, all the items were hid-

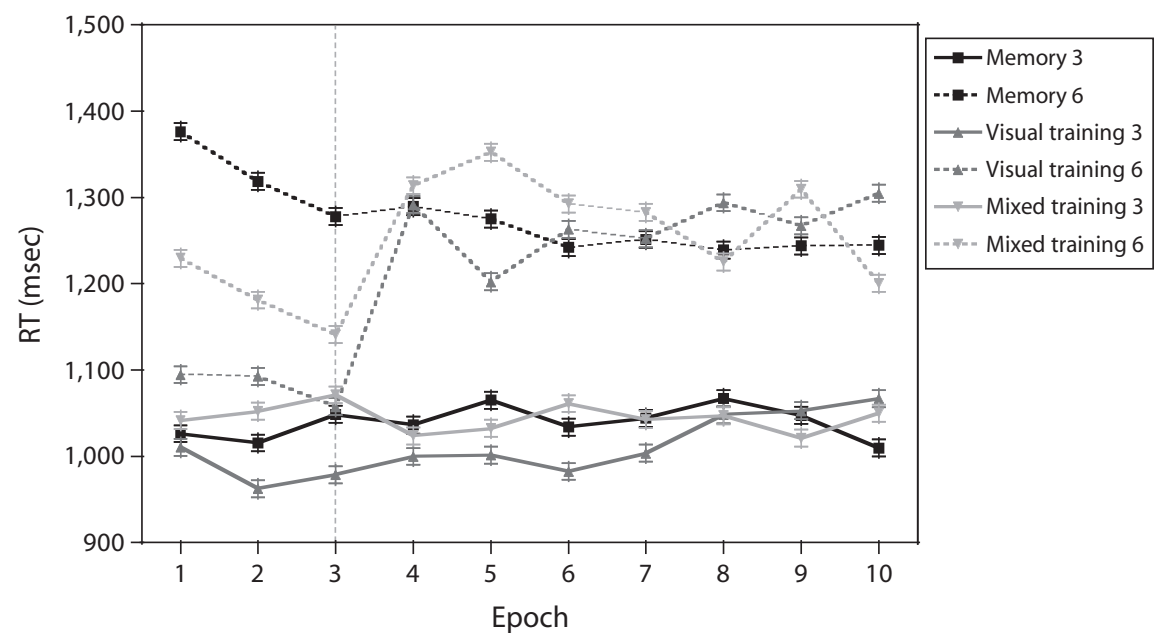

Figure 6. Reaction time (RT) as a function of epoch for the three conditions in Experiment 3. Note that during memory search, all three conditions produced essentially identical results. 


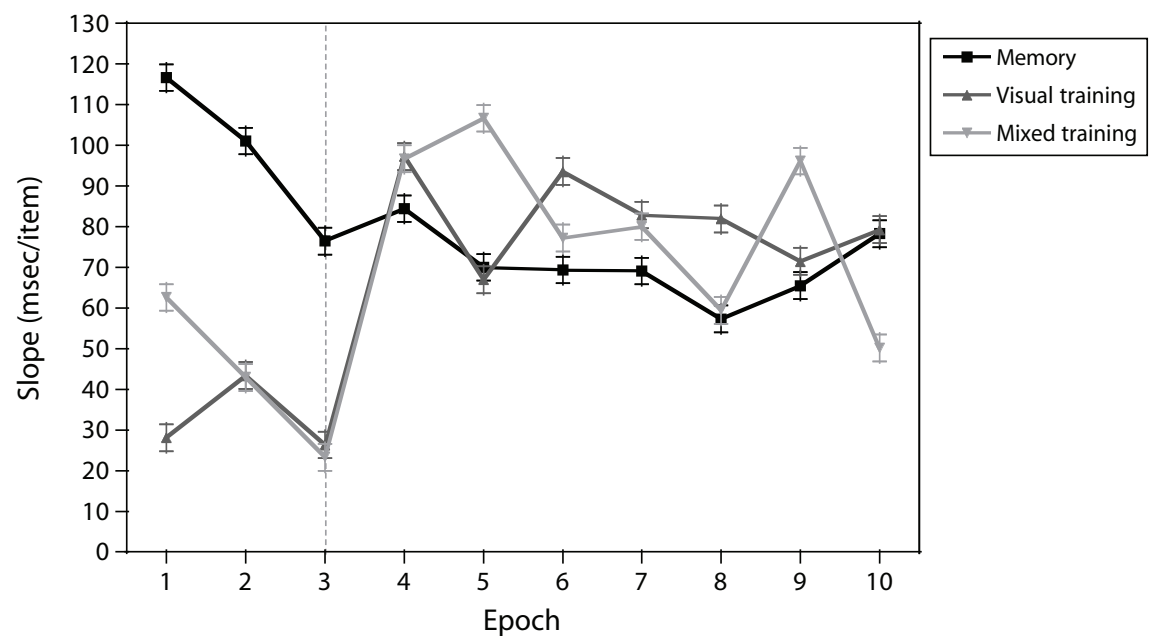

Figure 7. Slope as a function of epoch for the three conditions in Experiment 3. Note that during memory search, all three conditions produced essentially identical results.

den. Therefore, a previously visible stimulus had never been responded to by memory prior to the posttraining phase. Perhaps, if we switch whether each item was responded to by memory or vision during the initial training phase, search through memory will become more efficient. We investigated this in Experiment 4.

\section{EXPERIMENT 4}

\section{Method}

Participants. Twelve individuals between the ages of 18 and 55 years served as participants. Each participant passed the Ishihara test for color blindness and had normal or corrected-to-normal vision. All the participants gave informed consent and were paid for their time.

Apparatus and Stimuli. The apparatus and stimuli were identical to those in Experiment 2.

Procedure. There were three conditions: a pure memory search control condition and two training conditions - repeated visual search and a swap condition, in which a given search stimulus swapped from being hidden to visible or vice versa during the initial training period. In all the conditions the participants were asked to click on the location of the target. For each condition, the participants completed two blocks of trials, one each for set sizes 3 and 6 . The pure memory search and repeated visual search training conditions were identical to the conditions in Experiment 3. In the swap condition, the participants were instructed to memorize a search display prior to the task. The search display was identical to that in the repeated visual search training condition, except that each letter was surrounded by a box, defined by its white outline. At the start of the trial, a subset of the stimuli were removed, or hidden. This occurred for three of the stimuli when the set size was 6 and two of the letters when the set size was 3 . The remaining letters were visible, and the display remained unchanged for 15 trials. After Trial 15, the display changed so that the previously visible stimuli were removed and the previously hidden stimuli were now visible. This remained unchanged for the next 15 trials, at which point the visible stimuli became hidden and the hidden stimuli became visible (so that it resembled the first 15 displays). This process continued so that the visible/hidden status of each stimulus changed every 15 trials. After 150 trials (the initial training phase), all the letters were removed, so that none of the stimuli were visible, and the participants had to respond solely from memory. Each block consisted of 500 trials, which were divided into 10 epochs of 50 trials. The order of the blocks was randomized.

\section{Results and Discussion}

The results of Experiment 4 mirrored those of Experiment 3. Memory search in the posttraining phase was essentially the same with or without training.

Overall error rates were low $(1 \%)$. There was a main effect of condition $[F(2,22)=5.4, p<.05]$, where the participants made more errors in the memory condition than in the repeated search and swap conditions, and of set size $[F(1,11)=20.3, p<.01]$, where the participants made more errors for set size 6 than for set size 3 . There was also a reliable condition $\times$ epoch interaction $[F(18,198)=2.0, p<.05]$, where error rates increased in the repeated search condition between the training and the posttraining phases but did not change in the memory or swap condition. However, none of the other main effects or interactions proved reliable. Since the error rates suggest that there was no speed-accuracy trade-off, we will not discuss them further and, instead, will concentrate on RT and slope analysis. RTs below $200 \mathrm{msec}$ and above $4,000 \mathrm{msec}$ were eliminated. This led to the removal of $1 \%$ of the data.

For each condition, the data were split up into the initial training phase (Epochs 1-3) and the posttraining phase (Epochs 4-10) for analysis purposes. The data for the swap condition, in the initial training phase, were also separated into whether the target on a given trial was visible or hidden.

During the initial training period, there were reliable differences between the conditions in $\mathrm{RT}[F(3,33)=7.6$, $p<.01]$. RTs were shortest in the visual training condition, longest in the memory and hidden trials of the swap condition, and intermediate in the visible trials of the swap condition. There was a similar difference in slopes $[F(3,33)=7.7, p<.01]$. After training (Epochs $4-10)$, 
A Repeated Set Size 12
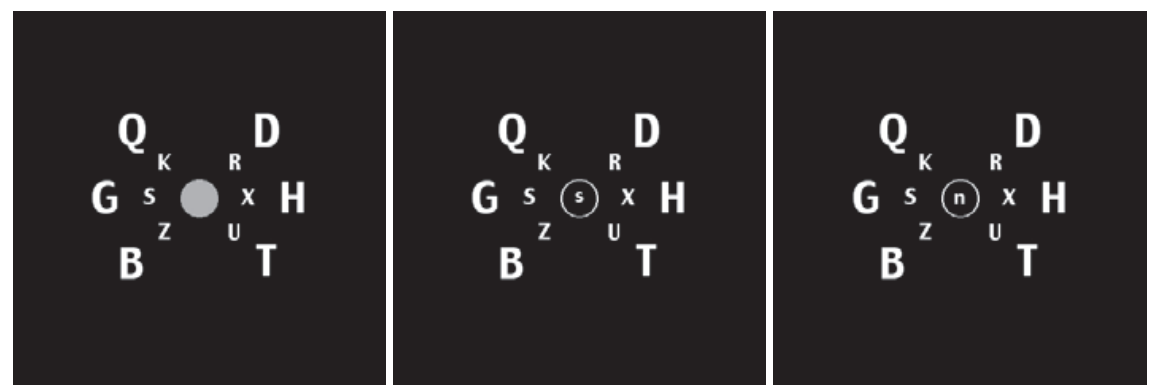

B Unrepeated Set Size 12
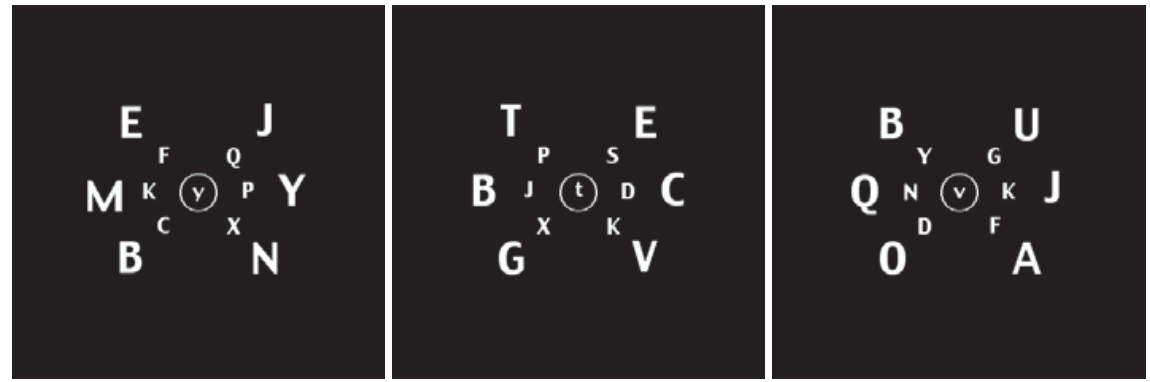

Figure 8. Example displays for Experiment 5. For the repeated search condition, the set size was blocked and could be $2,4,6,12$, or 18 . Here, the probe size equaled the set size when the set size was 2,4 , or 6 . However, when the set size was 12 or 18 , only 2,4 , or 6 items were probed. The unrepeated search set size was blocked and could be either 12 or 18 .

there were no significant differences in $\mathrm{RT}[F(2,22)=$ 0.7 , n.s. $]$ or slope $[F(2,22)=0.8$, n.s. $]$.

Overall, the results in the posttraining phase were similar to those in Experiment 3. Even in the swap condition, when all the items were trained as both visual and memory search targets, there was no benefit in the posttraining phase.

To summarize Experiments 1-4, when memory search cannot be reduced to a $2 \mathrm{AFC}$ response-mapping problem, it remains inefficient after hundreds of trials. This is true even if observers are given extended visual and/or mixed training prior to the memory search. The failure of observers to use memory of target locations during repeated visual search (Wolfe et al., 2000) is no longer a mystery. Relying on memory (e.g., look at this location to find that letter) is less efficient than simply rerunning the visual search, even if that visual search is inefficient.

As was noted at the outset, at face value, the results of repeated search experiments fly in the face of our common experience. When faced with familiar real-world scenes, we can often restrict our attention to the locations that we have learned to be relevant for the task in hand. Experiment 5 addressed this issue, showing that, if only a subset of target locations are ever relevant in repeated search, observers can use this information to restrict their search to this subset of potential targets and target locations.

\section{EXPERIMENT 5}

In Experiment 5, we investigated whether observers could restrict their search to a subset of repeated relevant locations. For example, if the participants learned that, within a display of $N$ items, only $M$ of them would ever be queried, could they learn to restrict their visual search to the relevant subset of those $M$ items?

\section{Method}

Participants. Thirteen naive observers between the ages of 18 and 55 years served as participants. Each participant passed the Ishihara test for color blindness and had normal or corrected-to-normal vision. All the participants gave informed consent and were paid for their time.

Apparatus and Stimuli. The search stimuli were similar to those in Experiment 1. Here, however, the search stimuli consisted of 2, 4, 6, 12, or 18 uppercase letters (omitting the letters "I" and "L"). All the letters were white and appeared on a black background. Because visual acuity declines as a function of distance from the fixation point, the size of the letters increased with eccentricity. Those closest to the center subtended a visual angle of $0.5^{\circ} \times 0.6^{\circ}$, whereas those farther out subtended a visual angle of either $1.0^{\circ} \times 1.2^{\circ}$ or $2.0^{\circ} \times 2.5^{\circ}$, depending on their eccentricity. All the stimuli were viewed from a distance of $57.4 \mathrm{~cm}$. The target probe was a lowercase letter presented at the center of the screen within a circle with a diameter of $1.5^{\circ}$.

Procedure. There were two conditions: a repeated search task and an unrepeated search task (see Figure 8 for example displays). In all the conditions, the participants had to decide whether the central target probe was present or absent from the display. The participants pressed a left key ("a") if it was present and a right key ("l") if it was absent and were asked to respond as quickly but as accurately as possible. The target probe changed on every trial, as in Experiments $1-4$.

In the repeated search task, the search array always remained on the screen. In each condition, there were $2,4,6,12$, or 18 items on the screen. For set sizes 2,4 , and 6 , all the stimuli could serve as targets (there was also an equal number of letters used as target-absent 
probes). This replicates Wolfe et al. (2000). For set sizes 12 and 18, only a subset of the letters in a display could serve as targets. Thus, although there were always 12 or 18 letters visible, only 2,4 , or 6 items were probed (again, there was an equal number of letters used as target-absent probes). For example, consider Figure 8A. The overall set size is 12 , but the participants might be queried only about the letters Q, S, T, or X. In this probe set size 4 condition, none of the other visible letters would ever be asked about. Four other letters (e.g., A, F, N, P) would be used as probes on target-absent trials. The letters used as targets remained constant throughout a block of trials, so that the participants learned by experience that only a subset of the letters and a subset of locations were relevant in each block. Since the physical display did not change, the identity and locations of the stimuli remained perfectly correlated for a block of trials. Thus, by constantly being probed about the same set of letters, the participants could learn that they needed to search only a subset of locations.

The unrepeated search task was intended as a baseline condition, to measure the rate of search through these stimuli under standard conditions. In this task, the search stimuli changed from trial to trial, along with the target probe. The set size was varied from 12 or 18 items, any of which could be a target, and thus all locations were being searched with equal probability. Each condition consisted of 350 experimental trials, and the participants completed a block of practice trials prior to the experiment proper. The order of blocks was randomized across participants.

\section{Results and Discussion}

The results for target-present trials are shown in Figures 9 and 10. The pattern of target-absent results was similar. The critical finding is that search varies with the probed set size, and not with the screen set size. For example, if only 4 items are probed, it does not matter if the physical set size is 4,12 , or 18 . Observers can restrict search to just these 4 items. Observers will need to search inefficiently through those 4, but not through the other 8 or 14 irrelevant items.
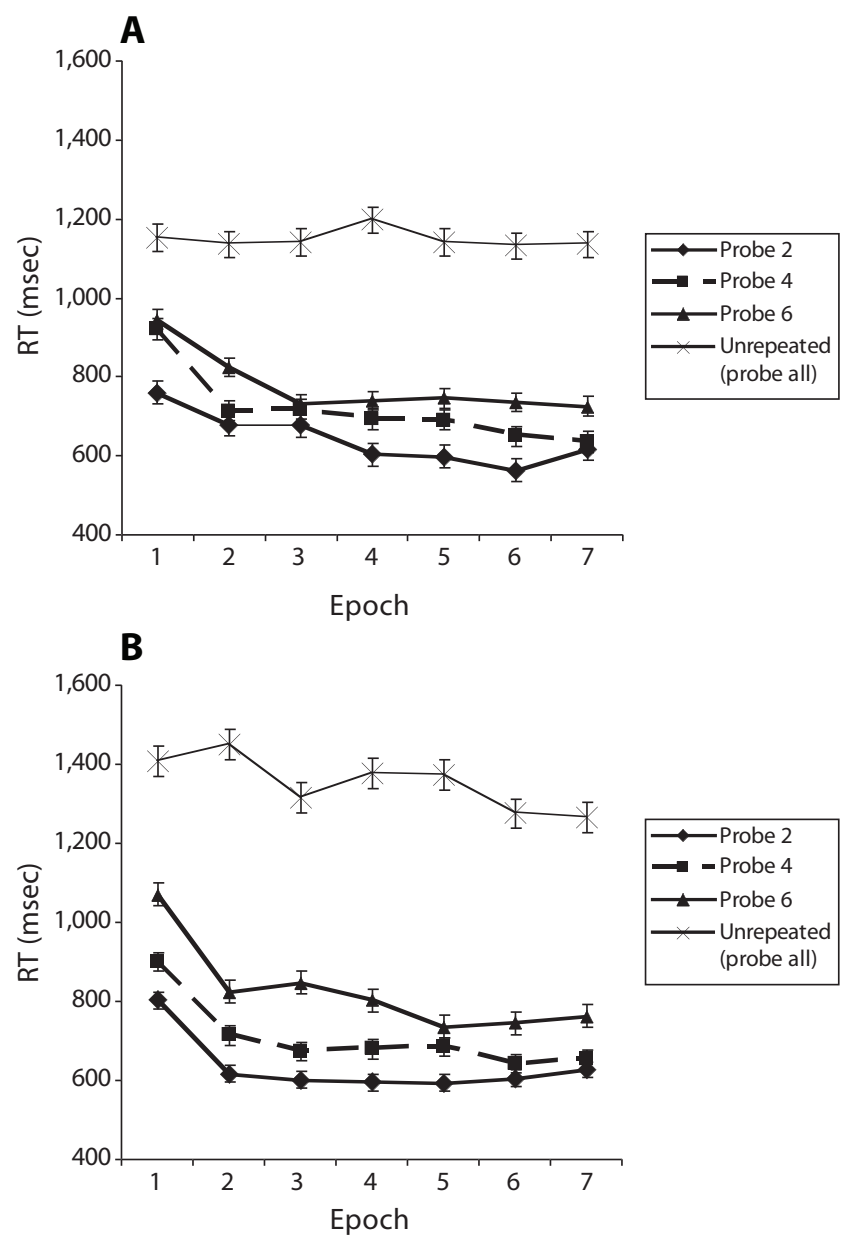

Figure 9. Reaction times (RTs) over epochs for the repeated (probe 2, probe 4, and probe 6) and unrepeated conditions in Experiment 5. The probe numbers refer to the number of locations that were searched in the repeated conditions. (A) Data when the overall physical set size was 12. (B) Data when the overall physical set size was 18 . RTs decreased over the first few epochs in the repeated conditions, indicating that the participants were learning to search only the relevant locations. 
Overall, error rates were quite low at 3\%. There was a main effect of target presence, with errors for targetpresent trials higher than those for target-absent trials $[F(1,12)=38.4, p<.01]$. Errors for unrepeated trials were higher than those in the memory and repeated conditions $[F(13,156)=9.9, p<.01]$. However, this was more pronounced for the target-present trials than for the targetabsent trials, as is shown by the target present/absent $\times$ condition interaction $[F(13,156)=19.7, p<.01]$. Since the error rates suggest that there was no speed-accuracy trade-off, we will not discuss them further and, instead, will concentrate on RT and slope analysis. RTs below $200 \mathrm{msec}$ and above 4,000 msec were eliminated. This led to the removal of fewer than $1 \%$ of the data.

Figure 9 shows the RT data across epochs for the display with the overall physical set size of 12 (Figure 9A) and that for the overall physical set size of 18 (Figure 9B). In all the conditions, the participants responded more quickly in the repeated search tasks, in which they were asked only about a subset of the probes and, thus, the locations, than in the unrepeated search tasks, where the target could appear at any location (all $F_{\mathrm{s}}>30.5, p \mathrm{~s}<.01$, and all $F_{\mathrm{s}}>75.7, p \mathrm{~s}<.01$, for physical set sizes 12 and 18 , respectively). RTs also decreased with epoch in the repeated conditions, suggesting that the participants were learning where the relevant target locations would be over the first few epochs $[F(6,72)=10.6, p<.01$, and $F(6,72)=15.1$, $p<.01$, for set sizes 12 and 18 , respectively].

For present purposes, the critical question is whether the observers learned to restrict search to the subset of locations that could contain a target. The clear answer was yes, as can be seen in Figures 10A and 10B. To look at asymptotic performance, we averaged the last 150 trials in each 350-trial block. In Figure 10A, we plot average targetpresent RTs as a function of the physical set size (12 or 18) for the three subset conditions (e.g., probe 2, probe 4, or probe 6 ), along with RTs from unrepeated search through 12 or 18 items. The probe-all condition replicates previous repeated search experiments, with search remaining inefficient after hundreds of trials. When targets were restricted to subsets of 2, 4, and 6, however, physical set size became irrelevant. Only the size of the relevant set mattered, as can be seen from the increase in RT for the subsets from 2 to 4 to 6 items. If the observers had not learned to restrict search to the relevant subset, all of the conditions plotted in Figure 10A should have been equivalent in efficiency (although performance in the repeated search conditions might have been somewhat faster than that in the unrepeated control conditions). Clearly, this was not the case. The unrepeated probe-all condition yielded a slope of $28 \mathrm{msec} /$ item that is highly different from $0 \mathrm{msec} /$ item $[t(12)=7.0, p<.01]$. For searches through subsets, the search slopes do not differ from $0 \mathrm{msec} /$ item (all $t \mathrm{~s}<1$, $p \mathrm{~s}>.6){ }^{2}$ Likewise, RTs in the repeated search conditions were shorter than those in the unrepeated condition (all $F \mathrm{~s}>49, p \mathrm{~s}<.01)$. In these repeated conditions, the participants were searching only the relevant items and were ignoring the rest of the distractors.
In Figure 10B, we plot target-present RTs as a function of the probed set size. When the physical set size was 2 , 4 , or 6 , the probed and physical set sizes were the same. When physical set size equaled 12 or 18 , the probed subset was 2, 4, or 6 items. In Figure 10B, we see that the repeated search subset conditions were equivalent to the standard repeated search conditions with physical set sizes of 2, 4, and 6. The slopes of $30.4 \mathrm{msec} /$ item (set sizes 2, 4, and 6), $35.8 \mathrm{msec} /$ item (set size 12), and $34.7 \mathrm{msec} /$ item (set size 18$)$ did not differ significantly $(F<0.2)$.

The important conclusion to be drawn from Experiment 5 is that participants are perfectly capable of restricting search to a relevant subset of stimuli in a repeated search task. However, in agreement with prior work and Experiments 1-4, it is not possible to eliminate search through limiting the set of relevant items. Whether the set of possible targets is the set of all items or a subset, observers search through that set with the same efficiency after 350 trials as they did before.

\section{EXPERIMENT 6}

In Experiment 6, we again investigated whether participants could learn to restrict their attention to a subset of relevant items. Here, however, instead of a present/absent 2AFC response task, a localization response task similar to that in Experiments 1-4 was used.
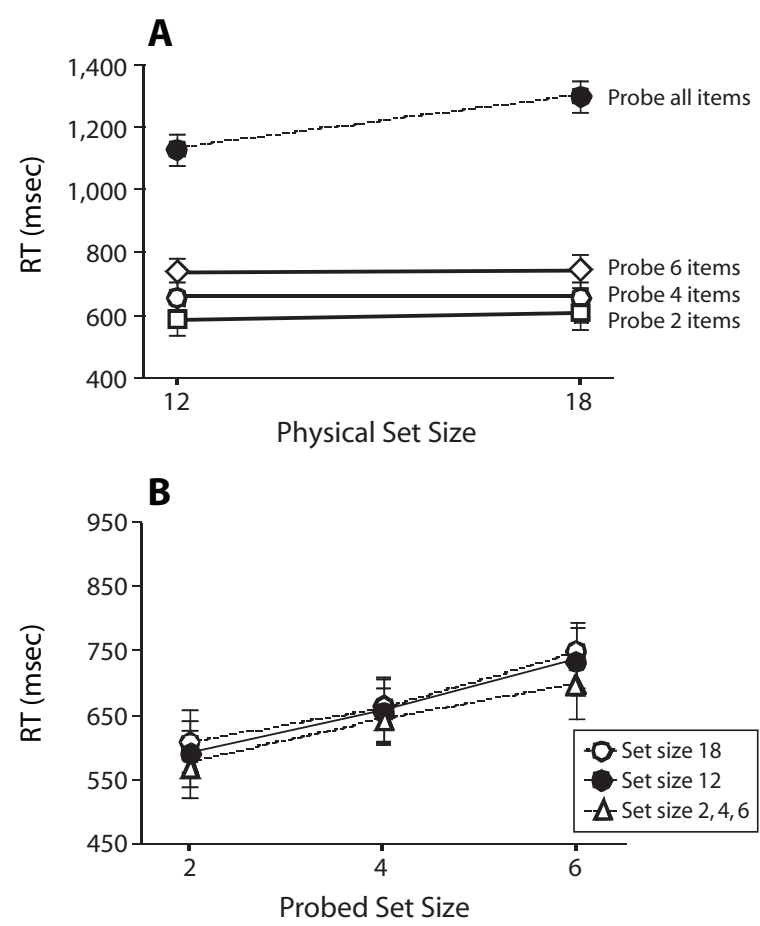

Figure 10. Reaction times (RTs) for the last 150 trials of 350-trial blocks in Experiment 5. (A) RT as a function of physical set size. (B) RT as a function of the number of items probed in the display. Clearly, it is probed set size that drives RT. 


\section{Method}

Participants. Twelve naive observers between the ages of 18 and 55 years served as participants. Each participant passed the Ishihara test for color blindness and had normal or corrected-to-normal vision. All the participants gave informed consent and were paid for their time.

Apparatus and Stimuli. The search stimuli were identical to those in Experiment 5.

Procedure. The procedure was similar to that in Experiment 5. However, in this case, the participants were asked to click on the target location if the target was present (as in Experiments 1-4). In addition to this, there were also trials on which the target was absent. In this case, the participants were asked to click on an orange square $\left(4^{\circ} \times 4^{\circ}\right)$ that was presented $15.7^{\circ}$ to the right of the central circle. In this experiment, the physical set size could be either 12 or 18 items. The probe set size (i.e., the number of items that were the only ones ever asked about) was fixed at either 3 or 6 . An equal number of let- ters were used as target-absent probes. Two unrepeated conditions (with set sizes 12 and 18) were used as baselines, in which the search display changed from trial to trial. Here, the target could be any item in the display and could thus appear in any position.

\section{Results and Discussion}

Data from 1 participant was not included in the analysis, since her data file was corrupted. The results for target-present trials are shown in Figures 11 and 12. The pattern of target-absent results was similar. Even when the response task was changed from a present/absent $2 \mathrm{AFC}$ task to a localization task, the results replicated those of Experiment 5. Search varied with the probed set size, and not with the screen set size, and thus could be restricted to a subset of relevant items.
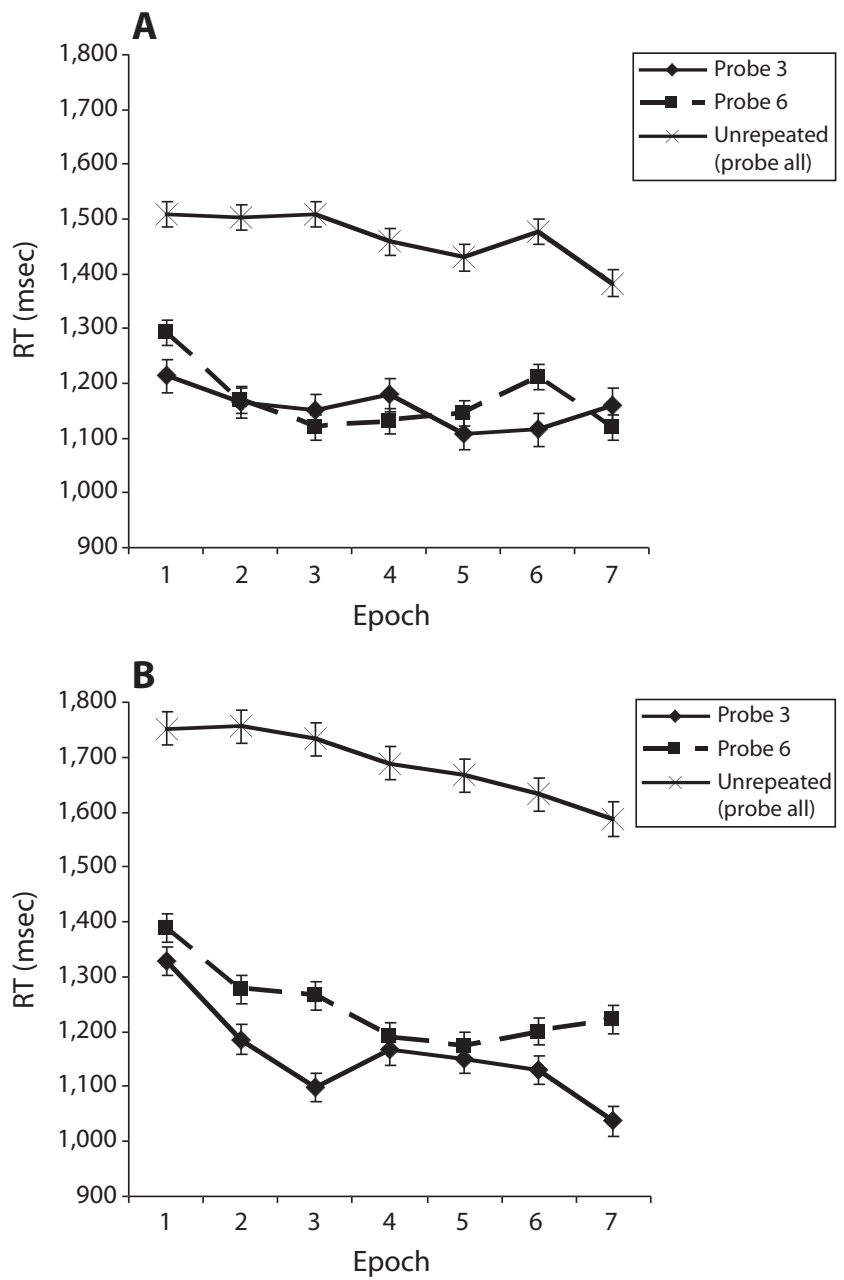

Figure 11. Reaction times (RTs) over epochs for the repeated (probe 3 and probe 6) and unrepeated conditions in Experiment 6. The probe numbers refer to the number of locations that were searched in the repeated conditions. (A) Data when the overall physical set size was 12 . (B) Data when the overall physical set size was 18. RTs decreased over the first few epochs in the repeated conditions, indicating that the participants were learning to search only the relevant locations. 


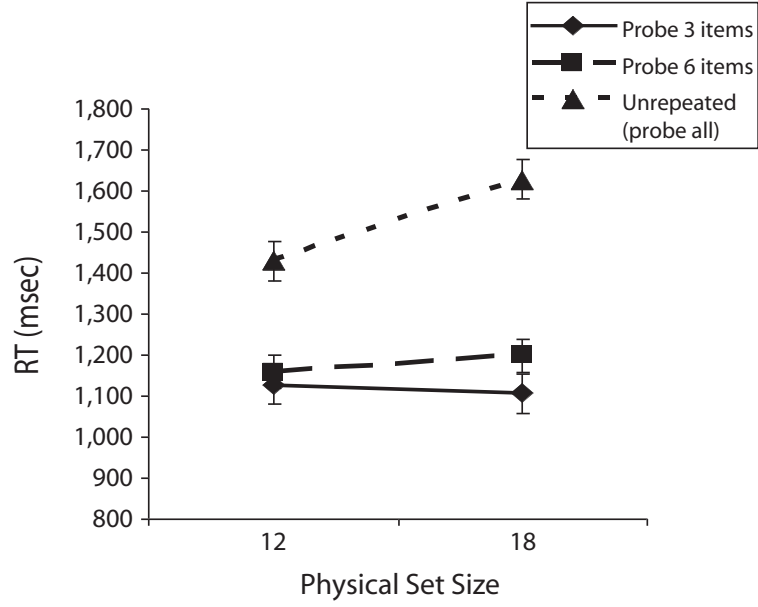

Figure 12. Reaction times (RTs) for the last 150 trials of 350-trial blocks in Experiment 6 as a function of physical set size.

Overall, error rates were quite low at 3\%. There was a main effect of condition $[F(1,10)=47.2, p<.01]$, where there was a higher percentage of errors in the unrepeated condition than in the repeated condition. Since the error rates suggest that there was no speed-accuracy trade-off, we will not discuss them further and, instead, will concentrate on RT and slope analysis. RTs below $200 \mathrm{msec}$ and above 4,000 msec were eliminated. This led to the removal of fewer than $2 \%$ of the data.

Figure 11 shows the RT data across epochs for the display with overall physical set size 12 (Figure 11A) and for that with overall physical set size 18 (Figure 11B). In all the conditions, the participants responded more quickly in the repeated search tasks, in which they were asked only about a subset of probes and, thus, locations, than in the unrepeated search tasks, in which the target could appear at any location (all $F \mathrm{~s}>22.1, p \mathrm{~s}<.01$, and all $F \mathrm{~s}>71.0$, $p \mathrm{~s}<.01$, for physical set sizes 12 and 18 , respectively). RTs also decreased with epoch in the repeated conditions, suggesting that the participants were learning where the relevant target locations would be over the first few epochs $[F(6,60)=3.6, p<.01$, and $F(6,60)=8.5, p<.01$, for set sizes 12 and 18 , respectively].

As in Experiment 5, the data again show that the observers learned to restrict search to the subset of locations that could contain a target. To look at asymptotic performance, we averaged the last 150 trials in each 350-trial block. In Figure 12, we plot average target-present RTs as a function of physical set size (12 or 18) for the two subset conditions (e.g., probe 3 or probe 6 ), along with RTs from the unrepeated search through 12 or 18 items. When targets were restricted to subsets of 3 and 6 in repeated search, physical set size became irrelevant. For repeated searches through subsets of both 3 and 6 items, the search slopes do not differ from $0 \mathrm{msec} /$ item $[t(10)=-0.4$, n.s., and $t(10)=1.4$, n.s., respectively]. This was not the case for the unrepeated search task, where search slopes showed inefficiency at $33 \mathrm{msec} /$ item and were much higher than $0 \mathrm{msec} /$ item $[t(10)=6.8, p<.01]$. Mirroring the results from Experiment 5, the data suggest that the participants were searching only the relevant items and were ignoring the rest of the distractors.

The findings from Experiment 6 replicate and extend those of Experiment 5. Again, the data show that participants are able to restrict their attention to a subset of relevant items within a repeated search task. The implications of this will be discussed further in the General Discussion section.

\section{GENERAL DISCUSSION}

To briefly summarize, repeating a visual search does not change the nature of that search. If the search is inefficient, it remains inefficient. The work presented here answers two puzzles within the repeated search literature. First, it explains why, even when participants can remember where stimuli are placed, they choose to search the display as though it were new. Experiments 1-4 showed that repeated memory search is less efficient than repeated visual search when observers are prevented from turning the memory search into a 2 AFC response-mapping task. Thus, given six possible targets in a repeated search task, observers do not use memory to instruct vision, because the memory search would be less efficient than simply rerunning the visual search. Second, Experiments 5 and 6 show that observers can restrict their attention to a subset of items if they learn that only these locations are ever relevant for search. Although search within the relevant subset remains inefficient, search performance overall is improved as participants learn to guide attention to specific locations and away from irrelevant stimuli. We conclude that the intuitively obvious improvement in search that occurs as a scene becomes familiar is due to this ability to restrict search to a subset of locations and is not due to a change in the nature of the underlying search.

It is possible that memory search might become efficient with enough repetition. Logan (1979) found that the consistent mapping of eight $\mathrm{S}-\mathrm{R}$ alternatives could be automatized if participants were trained extensively (e.g., over 6 days). This might have implications for some very overlearned visual search tasks (e.g., typing). However, in a world where S-R mapping may not be perfectly consistent and where stimuli are not continuously and exactly repeated, our data suggest that it will be more efficient to perform visual search than to rely on memory search.

This work is also important since it gives us insight into when context is important for search. Work in contextualcuing studies has shown that RTs to find a target are shorter when participants are shown a repeated display than when the display has not been seen before (e.g., Chun, 2000; Chun \& Jiang, 1998). On the face of it, the results of repeated search and contextual-cuing experiments might seem to be in conflict. ${ }^{3}$ In repeated search tasks, repeating a display does not improve search, whereas in contextualcuing tasks, RT, at least, improves. Research from our lab has shown that although RTs decrease with the number of repeated display repetitions, search slopes in contextual cuing do not show greater efficiency (Kunar, Flusberg, Horowitz, \& Wolfe, 2007; Kunar, Flusberg, \& Wolfe, 
2006). Thus, although participants respond more quickly when viewing a familiar context, a substantial portion of this effect may have nothing to do with improving the efficiency of search. A good portion of the contextual-cuing effect may be due to a facilitation of early or late processing components (such as response selection). Nevertheless, as our Experiments 5 and 6 show, there are circumstances in which the "context" of a repeated display can improve search efficiency by restricting search to a subset of the presented items.

\section{AUTHOR NOTE}

This research was supported by a grant from the National Institute of Mental Health to J.M.W. The authors thank Todd Horowitz and Kristin Michod for editorial advice and help with the experiments. Correspondence concerning this article should be addressed to M. A. Kunar, Department of Psychology, University of Warwick, Coventry CV4 7AL, England (e-mail: m.a.kunar@warwick.ac.uk).

\section{REFERENCES}

Brainard, D. H. (1997). The Psychophysics Toolbox. Spatial Vision, 10, 443-446.

Chun, M. M. (2000). Contextual cueing of visual attention. Trends in Cognitive Sciences, 4, 170-178.

Chun, M. M., \& Jiang, Y. (1998). Contextual cueing: Implicit learning and memory of visual context guides spatial attention. Cognitive Psychology, 36, 28-71.

Hick, W. E. (1952). On the rate of gain of information. Quarterly Journal of Experimental Psychology, 4, 11-26.

Hillstrom, A., \& Logan, G. D. (1998). Decomposing visual search: Evidence of multiple item-specific skills. Journal of Experimental Psychology: Human Perception \& Performance, 24, 1385-1398.

Hyman, R. (1953). Stimulus information as a determinant of reaction time. Journal of Experimental Psychology, 45, 188-196.

Kunar, M. A., Flusberg, S. J., Horowitz, T. S., \& Wolfe, J. M. (2007). Does contextual cueing guide the deployment of attention? Journal of Experimental Psychology: Human Perception \& Performance, 33, 816-828.

Kunar, M. A., Flusberg, S. J., \& Wolfe, J. M. (2006). Contextual cuing by global features. Perception \& Psychophysics, 68, 1204-1216.

KunAR, M. A., \& Humphreys, G. W. (2006). Object-based inhibitory priming in preview search: Evidence from the "top-up" procedure. Memory \& Cognition, 34, 459-474.

Kunar, M. A., Michod, K. O., \& Wolfe, J. M. (2005). When we use context in contextual cueing: Evidence from multiple target locations [Abstract]. Journal of Vision, 5(8), 412a.

LoGAN, G. D. (1979). On the use of a concurrent memory load to measure attention and automaticity. Journal of Experimental Psychology: Human Perception \& Performance, 5, 189-207.

LogAN, G. D. (1992). Attention and preattention in theories of automaticity. American Journal of Psychology, 105, 317-339.
Logan, G. D., Taylor, S. E., \& Etherton, J. L. (1999). Attention and automaticity: Toward a theoretical integration. Psychological Research, 62, 165-181.

Oliva, A., Wolfe, J., \& Arsenio, H. (2004). Panoramic search: The interaction of memory and vision in search through a familiar scene. Journal of Experimental Psychology: Human Perception \& Performance, 30, 1132-1146.

Pelli, D. G. (1997). The VideoToolbox software for visual psychophysics: Transforming numbers into movies. Spatial Vision, 10, 437-442.

SCHNeider, W., \& Shiffrin, R. M. (1977). Controlled and automatic human information processing: I. Detection, search and attention. Psychological Review, 84, 1-66.

STERNBERG, S. (1975). Memory scanning: New findings and current controversies. Quarterly Journal of Experimental Psychology, 27, 1-32.

Theeuwes, J., Kramer, A. F., \& Atchley, P. (1998). Visual marking of old objects. Psychonomic Bulletin \& Review, 5, 130-134.

Treisman, A. M., \& Gelade, G. (1980). A feature-integration theory of attention. Cognitive Psychology, 12, 97-136.

Treisman, A. [M.], \& Sato, S. (1990). Conjunction search revisited. Journal of Experimental Psychology: Human Perception \& Performance, 16, 459-478.

WoLfE, J. M. (1994). Guided Search 2.0: A revised model of visual search. Psychonomic Bulletin \& Review, 1, 202-238.

Wolfe, J. M. (1998). What do $1,000,000$ trials tell us about visual search? Psychological Science, 9, 33-39.

Wolfe, J. M., CAve, K. R., \& Franzel, S. L. (1989). Guided search: An alternative to the feature integration model for visual search. Journal of Experimental Psychology: Human Perception \& Performance, 15, 419-433.

Wolfe, J. M., Klempen, N., \& Dahlen, K. (2000). Postattentive vision. Journal of Experimental Psychology: Human Perception \& Performance, 26, 693-716.

\section{NOTES}

1. Overall, the memory slope for this experiment seems to show a greater efficiency than that for Experiment 1. One potential reason for this may be that memory search was affected by the context of having a prior visual search task. In this case, any previous consistent mapping within the visual search tasks may have added noise to the memory search task, making performance less efficient than when there were no other consistent-mapping tasks. We leave this for future work to investigate.

2 . These null results were not due to a lack of power. All of the analyses on these slopes show a power higher or equal to 0.9 , when looking for a similar difference to the unrepeated condition.

3. Please note that the repeated search paradigm and the contextualcuing paradigm share several differences, which may account in part for these apparently contradictory findings (see Kunar, Michod, \& Wolfe, 2005 , for details).

(Manuscript received December 6, 2005; revision accepted for publication September 10, 2007.) 